\title{
Detection of Plant Pathogenic Viruses in Commercial Gochujang (Fermented Red Pepper Paste) from Korea
}

\author{
Seoyeon Ko ${ }^{1}$, Na-Kyeong Kim ${ }^{1}$, Hyo-Jeong Lee ${ }^{1}$, Tae-Ho Ryu ${ }^{1}$, Jin-Sung Hong ${ }^{2 *}{ }^{2 *}$, and Rae-Dong Jeong (iD ${ }^{1 *}$ \\ ${ }^{I}$ Department of Applied Biology, Institute of Environmentally Friendly Agriculture, Chonnam National University, \\ Gwangiu 61185, Korea \\ ${ }^{2}$ Department of Applied Biology, College of Agriculture and Life Science, Kangwon National University, Chuncheon \\ 24341, Korea
}

(Received on June 1, 2020; Revised on June 22, 2020; Accepted on June 22, 2020)

The potential transmission of plant pathogenic viruses through processed foods could be a source of concern for global crop production; however, there is a lack of supporting evidence. The present study was conducted to investigate the presence of plant pathogenic viruses in five samples of gochujang (fermented red pepper paste) manufactured in Korea. Several viruses infecting pepper were detected by reverse transcriptionpolymerase chain reaction, among which the pepper mild mottle virus (PMMoV) was detected in all five samples, at concentrations ranging from 2.8 to $\mathbf{7 . 0}$ $\left(\log _{10}\right.$ copies $\left./ \mathrm{ml}\right)$. In addition, PMMoV was observed by transmission electron microscopy in all five samples. The samples exhibited viral pathogenicity to Nicotiana benthamiana plants, indicating that global trade of processed products could be a possible source of the transmission of plant viruses.

\footnotetext{
*Co-corresponding authors.

Rae-Dong Jeong

Phone) +82-62-530-2075, FAX) +82-62-530-2069

E-mail) jraed2@jnu.ac.kr and

Jin-Sung Hong

Tel) +82-33-250-6437, FAX) +82-33-259-5558

E-mail) jinsunghong@kangwon.ac.kr

ORCID

Rae-Dong Jeong

https://orcid.org/0000-0002-6370-8909

Jin-Sung Hong

https://orcid.org/0000-0001-5011-9999

(c) This is an Open Access article distributed under the terms of the Creative Commons Attribution Non-Commercial License (http:// creativecommons.org/licenses/by-nc/4.0) which permits unrestricted noncommercial use, distribution, and reproduction in any medium, provided the original work is properly cited.
}

Articles can be freely viewed online at www.ppjonline.org.
Keywords : detection, Gochujang, plant viruses

Handling Editor : Ju-Yeo Yoon

Red pepper (Capsicum annuum L.) is one of the most widely used spices in the world and is mainly utilized in the dried powder form. In Korea, red pepper powder is used to prepare a sauce or paste for ethnic foods (Kwon et al., 2015). Gochujang (fermented red pepper paste), a traditional Korean sauce, is a savory, sweet, and spicy fermented condiment prepared from red pepper powder, meju (a mixture of starch and wheat grain), and water. It consists of $44.6 \%$ water, $43.8 \%$ carbohydrates, $4.9 \%$ protein, and $1.1 \%$ fat, which are produced from the raw materials during fermentation (Kim et al., 2016). Furthermore, gochujang contains major bioactive compounds, such as carotenoids, capsaicinoids, and flavonoids, and it stimulates several biological functions owing to its anti-cancer, anti-obesity, and anti-oxidant effects (Ahn et al., 2006; Park et al., 2001).

Red pepper powder is one of the raw materials for the production of commercial gochujang. This powder is prepared from fresh red peppers, which may have been exposed to various plant pathogens, particularly to plant viruses. Food products containing red peppers or spices, such as gochujang, could be potential sources of viral ingestion. Of the 66 viruses known to infect peppers, 29 occur worldwide, whereas 37 occur regionally. Five major viruses, i.e., cucumber mosaic virus (CMV), broad bean wilt virus 2 (BBWV2), pepper mottle virus (PepMoV), pepper mild mottle virus (PMMoV), and tomato spotted wilt virus (TSWV), infect red peppers in Korea (Cho et al., 2006; Choi et al., 2010; Kim et al., 2004). The aim of the present study was to detect pathogenic plant viruses and reveal their molecular genetic characterization in five major 
commercial gochujang products manufactured in Korea.

Five major commercial gochujang products $(\mathrm{H}, \mathrm{CH}, \mathrm{HP}$, $\mathrm{J}$, and S), manufactured in 2018 and 2019, were purchased from commercial markets. The red pepper powders used in these products were derived from either Korean fresh red peppers $(\mathrm{H}$ and $\mathrm{CH})$ or frozen red peppers $(\mathrm{HP}, \mathrm{J}$, and S) imported from China. Red pepper powder derived from fresh red peppers is the major component in the production of commercial gochujang. To investigate the presence of plant viruses in gochujang samples, we first analyzed the virus particles using transmission electron microscopy (TEM). Gochujang was mixed gently with $1 \times$ phosphate buffered saline (PBS) buffer (1:1), and the mixture was kept at room temperature for $10 \mathrm{~min}$. Mixture was centrifuged at 12,000 rpm for $20 \mathrm{~min}$. The supernatant was then used for TEM analysis. Samples were fixed on a copper grid (Electron Microscopy Sciences, Hatfield, PA, USA) and negatively stained with $1 \%$ ammonium molybdate (Sigma-Aldrich, St. Louis, MO, USA). Fixed samples were analyzed using a TEM (JEOL Ltd., Tokyo, Japan) at 200 $\mathrm{kV}$, at the Center for Research Facilities of Chonnam National University. For each grid, at least three fields of ap- proximately $200 \mu \mathrm{m}^{2}$ were viewed. Images were captured using a US1000 CCD camera and analyzed using GATAN MICROGRAPH software (Gatan Inc., Warrendale, PA, USA). All samples contained rod-shaped particles, measuring $300 \mathrm{~nm}$ in length and $18 \mathrm{~nm}$ in diameter, which were identified as tobamoviruses (Fig. 1A). Virus-free gochujang confirmed by TEM and reverse transcriptionpolymerase chain reaction (RT-PCR) was used as negative control. Furthermore, RT-PCR was performed to detect the five major viruses infecting red peppers in Korea, i.e., CMV, BBWV2, PepMoV, PMMoV, and TSWV. Total RNA was extracted from the samples using TRIzol reagent (Invitrogen, Carlsbad, CA, USA), according to the manufacturer's instructions. In brief, $300 \mu$ of gochujang was gently mixed with $1 \mathrm{ml}$ of TRIzol, and mixture was kept at room temperature for $10 \mathrm{~min}$. Two hundred microliters of chloroform was added and incubated for $5 \mathrm{~min}$. The suspensions were followed centrifugation at $12,000 \mathrm{rpm}$ for $10 \mathrm{~min}$. The supernatant was precipitated with isopropanol, dissolved with DEPC-water. The prepared total RNA was stored at $-80^{\circ} \mathrm{C}$ until processing. A one-tube RT-PCR was performed using a SuPrimeScript RT-PCR Premix (GeNet
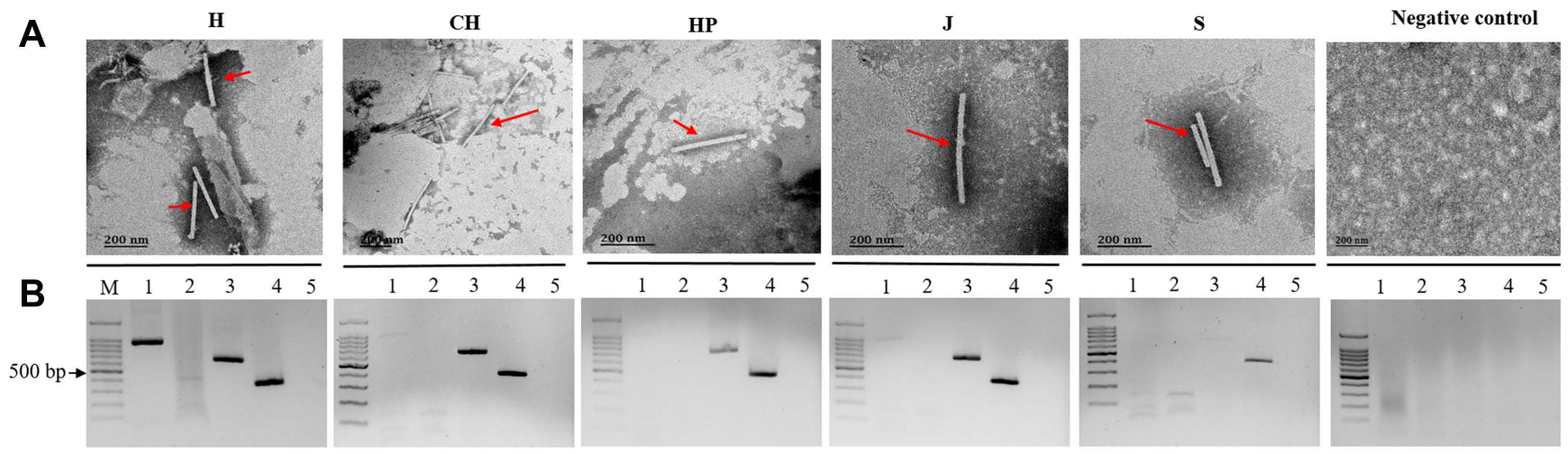

C
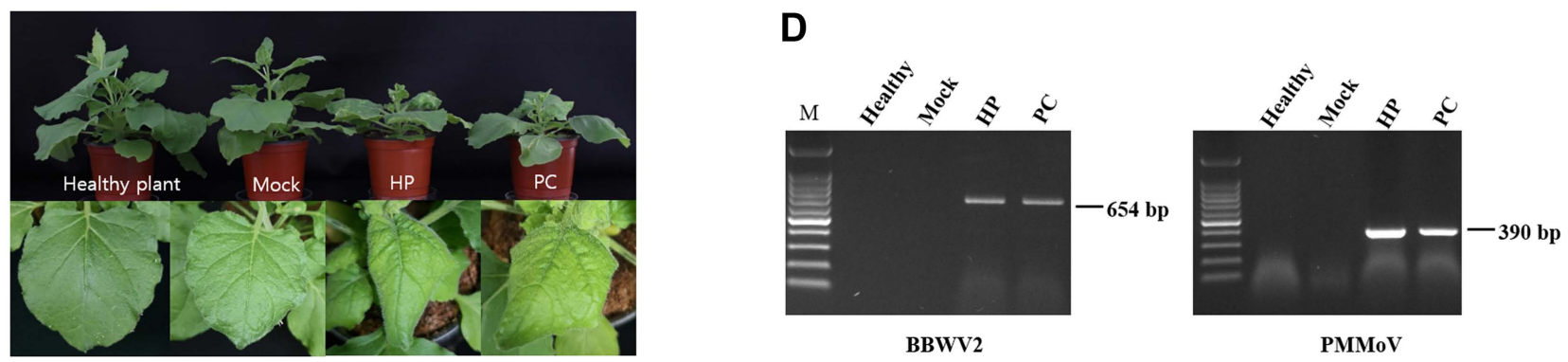

Fig. 1. Detection of plant viruses in gochujang samples. (A) Rod-shaped particles in the five samples observed by electron microscopy. (B) Agarose gels showing the expected sizes of PCR products of the target viruses. M, $1 \mathrm{~kb}$ DNA ladder; lane 1, cucumber mosaic virus (CMV; 940 bp); lane 2, pepper mottle virus (PepMoV; 461 bp); lane 3, broad bean wilt virus 2 (BBWV2; 654 bp); lane 4, pepper mild mottle virus (PMMoV; 390 bp); lane 5, tomato spotted wilt virus (TSWV; 500 bp). (C) Symptoms induced in Nicotiana benthamiana by the gochujang-HP sample at 14 dpi. The upper panel shows plants in the side-view, and the lower panel shows an enlarged top view. PC, positive control. (D) Validation of systemic infection of BBWV2 and PMMoV in the HP-treated and positive control plants by reverse transcription-polymerase chain reaction. 
Bio, Daejeon, Korea). Two microliters of total RNA template, and forward and reverse primers for CMV, BBWV2, PepMoV, PMMoV, and TSWV were added to the RTPCR premix, and DEPC water was then added to achieve a total volume of $20 \mu$ l. Coat protein (CP)-specific primers of the pepper viruses were used for their detection in the samples (Supplementary Table 1). The conditions for RT-PCR were as follows: stage $1,50^{\circ} \mathrm{C}$ for $30 \mathrm{~min}$; stage $2,95^{\circ} \mathrm{C}$ for $5 \mathrm{~min}$; stage 3,38 cycles each of $95^{\circ} \mathrm{C}$ for $30 \mathrm{~s}, 58^{\circ} \mathrm{C}$ for 30 $\mathrm{s}$, and $72^{\circ} \mathrm{C}$ for $60 \mathrm{~s}$; and stage $4,72^{\circ} \mathrm{C}$ for $5 \mathrm{~min}$. The PCR products were analyzed by $1.2 \%$ agarose gel electrophoresis in $1 \times$ TAE buffer, stained with ethidium bromide, and visualized under UV light. The size of the resultant product was determined by comparison with a 100 bp DNA ladder (iNtRON, Daejeon, Korea). The predicted fragment of the $\mathrm{PMMoV}$ genome was amplified in all the five gochujang samples (H, CH, HP, J, and S) (Fig. 1B). The predicted fragments of the BBWV2 and PMMoV genomes could be amplified in the $\mathrm{H}, \mathrm{CH}, \mathrm{HP}$, and $\mathrm{J}$ samples. In the $\mathrm{H}$ sample, the predicted fragment of the CMV genome was also amplified along with that of BBWV2 and PMMoV. In addition, the predicted fragment of only the PMMoV genome was amplified in the $\mathrm{S}$ sample. The presence of $\mathrm{PMMoV}$ was recently reported in pepper sauce from China (Peng et al., 2015). Furthermore, PMMoV was detected in all 42 pepper sauce samples from China, using RT-PCR. To determine whether the detected viruses in these samples were infectious, gochujang samples $(\mathrm{H}, \mathrm{CH}, \mathrm{HP}, \mathrm{J}$, and $\mathrm{S})$ were mixed with PBS, and after centrifugation at 12,000 rpm for $10 \mathrm{~min}$ at $4^{\circ} \mathrm{C}$, the supernatant was collected and used to inoculate healthy $N$. benthamiana indicator plants. Virus-free gochujang was used as a negative control, and virus-infected leaf samples were used as a positive control. Plants inoculated with $\mathrm{H}, \mathrm{CH}, \mathrm{J}$, and $\mathrm{S}$ samples exhibited mild mosaic or mottle symptoms in inoculated and systemic leaves at $14 \mathrm{dpi}$, and the presence of viruses was confirmed by RT-PCR using the aforementioned primers (Supplementary Fig. 1). Plants inoculated with HP samples exhibited leaf wilting and stem necrosis (Fig. 1C). Positive control plants co-inoculated with BBWV2 and PMMoV purified from the infected plants presented symptoms similar to plants inoculated with the HP sample, whereas the mock-inoculated plants did not exhibit any obvious symptoms (Fig. 1C). RT-PCR analysis showed that plants inoculated with the HP sample and the positive controls were systemically infected with BBWV2 and PMMoV, whereas the mock-inoculated plants did not present any viruses (Fig. 1D). These results indicated that BBWV2 and PMMoV in the gochujang samples retained their infectivity in plants.

The amplified RT-PCR products were cloned and se- quenced to confirm the identity of the viruses. The RTPCR products were purified using the MEGAquick-spin Plus Fragment DNA Purification Kit (iNtRON) and cloned using pGEM-T Easy Vector System (Promega, Madison, WI, USA), following which the clones were sequenced in both directions (Bionics Co., Daejeon, Korea). The sequences obtained were compared with the reference sequences for virus isolates from the GenBank database. Nucleotide sequences of the complete sequence of the CMV$\mathrm{CP}$ and the partial CP of PepMoV, BBWV2, PMMoV, and TSWV2 have been deposited in the GenBank database (LC528236: CMV isolate from H sample; LC534249LC534252: BBWV2 isolates from $\mathrm{H}, \mathrm{CH}, \mathrm{HP}$, and $\mathrm{J}$ samples; LC528237-LC528241: PMMoV isolates from $\mathrm{H}, \mathrm{CH}, \mathrm{HP}, \mathrm{J}$, and $\mathrm{S}$ samples). For phylogenetic analysis, nucleotide sequences of virus isolates were aligned using CLUSTALW in BioEdit. Aligned nucleotide sequences were used to construct phylogenetic trees using MEGA 5.05 software package (Tamura et al., 2011). Bootstrap resampling (with 1,000 replications) was used to measure the reliability of the individual nodes in each phylogenetic tree. A neighbor-joining phylogenetic analysis was conducted to determine the relationship of CMV, BBWV2, and PM$\mathrm{MoV}$ isolates obtained from the gochujang samples with the previously characterized isolates of various geographic origins. Sequence and phylogenetic analyses revealed that the CMV-H (LC528236) sequence had 97-99\% nucleotide similarity, and the phylogenetic tree exhibited two groups (Fig. 2A). The first group was further divided two subgroups: one of these sub-groups comprised the Chinese and Korean isolates, including $\mathrm{CMV}-\mathrm{H}$, whereas the other sub-group included two isolates from China and three isolates from Korea. The second group comprised four isolates from Korea. Although the red pepper powder used in Kochujang-H was derived from Korean fresh powder, the CMV-H was confirmed to be close to the Chinese isolates. The BBWV2 isolates, i.e., BBWV2-H, BBWV2CH, BBWV2-HP, and BBWV2-J (LC534249-LC534252), exhibited $85-96 \%$ nucleotide similarity with the Chinese, Korean, and Japanese BBWV2 isolates. The four isolates were divided into two groups (Fig. 2B). The first group was further divided into two sub-groups: one of the sub-groups comprised five isolates from Korea, including BBWV2-J, whereas the other sub-group consisted of one isolate from Japan, one from China, and two from Korea, including BBWV2-HP. The second group was also divided into two sub-groups: BBWV2-H alone represented one of these subgroups, whereas the other sub-group included one isolate from Singapore, one from Japan, one from China, and four from Korea, including BBWV2-CH. Although the red pep- 

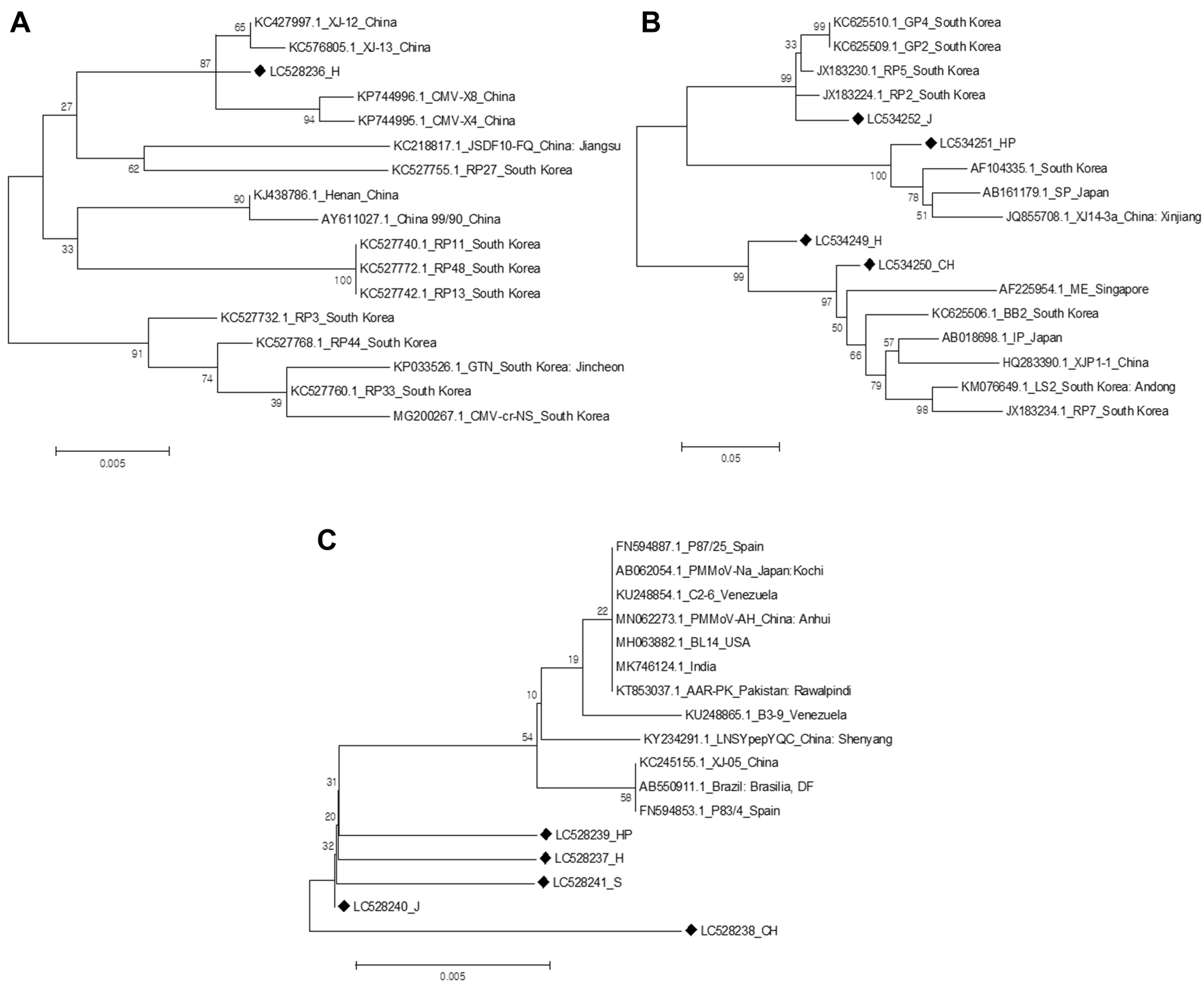

Fig. 2. Phylogenetic analysis of cucumber mosaic virus (CMV) (A), broad bean wilt virus 2 (BBWV2) (B), and pepper mild mottle virus $(\mathrm{PMMoV})(\mathrm{C})$ isolates detected in the gochujang samples. The neighbor-joining phylogenetic tree was constructed using coat protein sequences of isolates from different countries obtained from NCBI, using MEGA 6. The GenBank accession code, isolate name, and country of origin are shown for each isolate. Isolates described in this study are indicated by a diamond. Branch lengths are proportionate to genetic distances. The bootstrap analysis employed a heuristic search with 1,000 random stepwise additions of replicates. The scale bar represents 0.005 or 0.05 substitution per nucleotide site.

per powder used in Kochujang-J was derived from Chinese frozen powder, the BBWV2-J was confirmed to be close to the Korean isolates. The PMMoV isolates, i.e., PMMoV$\mathrm{H}, \mathrm{PMMoV}-\mathrm{CH}, \mathrm{PMMoV}-\mathrm{HP}$, PMMoV-J, and PMMoV-S (LC528237-LC528241), exhibited 98-99\% nucleotide similarity with isolates from China, Japan, USA, India, Brazil, Venezuela, and Pakistan. Phylogenetic analysis revealed that PMMoV isolates from the HP, S, H, and J samples and those from other countries were clustered in one group, whereas PMMoV-CH represented a separate group (Fig. $2 \mathrm{C})$. Although the red pepper powders used in Kochujang products $(\mathrm{H}, \mathrm{CH}, \mathrm{HP}, \mathrm{J}$, and $\mathrm{S})$ showed different production site of red pepper, the newly isolated PMMoV-HP, $\mathrm{H}$, and $\mathrm{S}$ isolates were clustered in one group. Phylogenetic analysis of the newly isolated viruses indicated no correlation between the geographic origin and groups of isolates. To quantify BBWV2 and PMMoV genomic RNAs, transcripts were obtained from biologically active cDNA clones of BBWV2 and PMMoV using T7 RNA polymerase (Promega) in vitro. The copy numbers were determined using the equation $N=C /\left(K \times 330 \times 1.6601 \times 10^{-18}\right)$, where $N$ is the copy number per $\mu$ l, $K$ is the length of the target gene 
Table 1. Detection of viruses in pepper sauce samples

\begin{tabular}{|c|c|c|c|c|c|c|c|}
\hline \multirow{2}{*}{ Sample } & \multicolumn{5}{|c|}{ Virus } & \multicolumn{2}{|c|}{ Concentration of viruses ${ }^{\mathrm{a}}$} \\
\hline & CMV & PepMoV & BBWV2 & PMMoV & TSWV & BBWV2 & PMMoV \\
\hline $\mathrm{H}$ & + & - & + & + & - & $6.01 \pm 0.0921$ & $6.50 \pm 0.1768$ \\
\hline $\mathrm{CH}$ & - & - & + & + & - & $3.84 \pm 0.0363$ & $4.94 \pm 0.1053$ \\
\hline HP & - & - & + & + & - & $3.11 \pm 0.0402$ & $7.00 \pm 0.3800$ \\
\hline $\mathrm{J}$ & - & - & + & + & - & $4.31 \pm 0.3220$ & $6.21 \pm 0.0078$ \\
\hline S & - & - & - & + & - & & $2.82 \pm 0.0826$ \\
\hline
\end{tabular}

CMV, cucumber mosaic virus; PepMoV, pepper mottle virus; BBWV2, broad bean wilt virus 2; PMMoV, pepper mild mottle virus; TSWV, tomato spotted wilt virus.

${ }^{a}$ The concentration of BBWV2 and PMMoV as $\log _{10}$ copies/ml (mean \pm SD)

(nucleotide), and $1.6601 \times 10^{-18}$ represents the transfer constant between dalton and $\mu$ g. Quantitative RT-PCR (RTqPCR) was performed to determine the concentrations of BBWV2 and PMMoV in the gochujang samples. Primers specific to BBWV2 and PMMoV were designed for RTqPCR, based on the respective $\mathrm{CP}$ genes of the viruses, using Primer3 Input (ver. 0.4.0) (Supplementary Table 1). The concentrations of BBWV2 and PMMoV in the gochujang samples were determined according to previously published methods (Feng et al., 2006). For RT-qPCR, cDNA was synthesized using each of the reverse primers and $\mathrm{M}$ MLV RT (Promega), according to the manufacturer's instructions. RT-qPCR reactions for BBWV2 and PMMoV were carried out in a final volume of $25 \mu 1$, containing $12.5 \mu \mathrm{l}$ of $2 \times$ QuantiFast SYBR Green PCR Master Mix (Qiagen, Valencia, CA, USA), $1.0 \mu 1$ each of the forward and reverse primers, and $1 \mu \mathrm{l}$ of the cDNA. Thermocycling conditions consisted of an initial denaturation step at $95^{\circ} \mathrm{C}$ for $5 \mathrm{~min}$, followed by 35 cycles of $95^{\circ} \mathrm{C}$ for $15 \mathrm{~s}, 56^{\circ} \mathrm{C}$ for $20 \mathrm{~s}$, and $60^{\circ} \mathrm{C}$ for $20 \mathrm{~s}$. Immediately after the final PCR cycle, a melting curve analysis was performed to determine the specificity of the reaction, by incubating the reaction mixture at $95^{\circ} \mathrm{C}$ for $15 \mathrm{~s}$ followed by annealing at $60^{\circ} \mathrm{C}$ for $20 \mathrm{~s}$. The $C_{t}$ used in RT-qPCR quantification was defined as the PCR cycle number that crossed an arbitrarily chosen signal threshold in the log phase of the amplification curve. Standard curves for all viruses were generated using a dilution series of standard samples as the RT-qPCR template. The extracted RNA was diluted 100 times and amplified along with standard samples under optimal concentrations, and the copy number of each virus was calculated from the standard curve according to the $C_{t}$ value. Each sample had three replicates, and all reactions were also performed in three independent replicates to ensure the reproducibility of the results. Concentrations of BBWV2 and PMMoV in gochujang samples ranged from 3.84 to $6.01 \log _{10}$ copies/ml and from 2.82 to $7.00 \log _{10}$ copies $/ \mathrm{ml}$, respectively
(Table 1). The results showed that the $\mathrm{H}$ sample contained a higher concentration of BBWV2 than the other samples, whereas the HP sample contained a higher concentration of PMMoV. These results are similar to those obtained for Chinese pepper sauce $\left(\approx 3.83\right.$ to $8.75 \log _{10}$ copies $/ \mathrm{ml}$ ) (Peng et al., 2015).

Few studies have described the presence of plant viruses in processed foods and human and wastewater samples (Peng et al., 2015; Symonds et al., 2019; Zhang et al., 2006). PMMoV was detected in pepper sauce samples from China, at concentrations ranging from 3.8 to $8.8 \log _{10}$ copies $/ \mathrm{ml}$. The CP sequences of PMMoV exhibited 97.4$100 \%$ similarity to each other and $92.4-100 \%$ similarity to previously characterized isolates. In addition, pepper sauce containing PMMoV caused viral symptoms in plants, indicating that commercial trade of processed sauce could be a source of natural dispersal of PMMoV (Peng et al., 2015). Globalization of the food sector has led to international trading of numerous processed products. This can result in the transport and outbreak of pathogenic plant viruses in other regions of the world. The issue of plant viruses, including the presence and pathogenicity of PMMoV in humans, was highlighted by the metagenomics discovery that plant viruses were the most abundant RNA viruses in human feces (Zhang et al., 2006). Up to $10^{9}$ virions of PM$\mathrm{MoV}$ were observed per gram dry weight of fecal matter, and PMMoV caused viral symptoms in indicator plants. In addition, using metagenomics, tobamoviruses were detected in fecal samples from Japan (Nakamura et al., 2009). These viruses infect various crops, including peppers (Capsicum spp.), and their presence in human feces may be a result of consuming processed products prepared from infected crops. Recently, PMMoV was also consistently detected in untreated wastewater from Africa, the Americas, Asia, Australia, and Europe; thus, it is often used as a viral indicator to identify fecal pollution in the environment (Kitajima et al., 2018; Symonds et al., 2019). 
In conclusion, this is the first study in which several pepper-infecting viruses were detected in commercial gochujang samples manufactured in Korea. Although the virus-infected red peppers used for the production of commercial gochujang were industrially processed, the identified viruses remained pathogenic to plants, highlighting the potential risk of a viral disease outbreak. Phylogenetic analysis indicated that the identified virus isolates were the most closely related to their Chinese counterparts, which may be attributed to the fact that the red pepper powder imported from China is often used in the production of gochujang. Although plant viruses do not directly threaten human health, the risk of spreading these viruses to other countries/regions through processed foods affects agricultural profitability, food security, and ecosystem sustainability. Further research is required to investigate the plant virome in other processed foods, as it would improve our understanding of the correlations between plant viruses derived from processed foods and the agri-environment.

\section{Acknowledgments}

This research was finally supported by Chonnam National University (Grant No. 2016-2498) and Cooperative Research Program for Agricultural Science \& Technology Development (Project No. PJ014507032020).

\section{Electronic Supplementary Material}

Supplementary materials are available at The Plant Pathology Journal website (http://www.ppjonline.org/).

\section{References}

Ahn, I. S., Do, M. S., Kim, S. O., Jung, H. S., Kim, Y. I., Kim, H. J. and Park, K. Y. 2006. Antiobesity effect of Kochujang (Korean fermented red pepper paste) extract in 3T3-L1 adipocytes. $J$. Med. Food 9:15-21.

Cho, J.-D., Lee, S.-H., Kim, J.-S., Choi, G.-S., Kim, H.-R., Chung, B.-N. and Ryu, K.-H. 2006. Characteristics of $\mathrm{Cu}-$ cumber mosaic virus-VCH causing vein chlorosis on red pepper in Korea. Res. Plant Dis. 12:226-230 (in Korean).
Choi, H. S., Lee, S. H., Kim, M. K., Kwak, H. R., Kim, J. S., Cho, J. D. and Choi, G. S. 2010. Occurrence of virus diseases of major crops in 2009. Res. Plant Dis. 16:1-9 (in Korean).

Feng, J.-L., Chen, S.-N., Tang, X.-S., Ding, X.-F., Du, Z.-Y. and Chen, J.-S. 2006. Quantitative determination of cucumber mosaic virus genome RNAs in virions by real-time reverse transcription-polymerase chain reaction. Acta Biochim. Biophys. Sin. 10:669-676.

Kim, J.-H., Choi, G.-S., Kim, J.-S. and Choi, J.-K. 2004. Characterization of Tomato spotted wilt virus from paprika in Korea. Plant Pathol. J. 20:297-301.

Kim, S.-H., Chung, K. R., Yang, H.-J. and Kwon, D. Y. 2016. Sunchang gochujang (Korean red chili paste): the unfolding of authenticity. J. Ethn. Foods 3:201-208.

Kitajima, M., Sassi, H. P. and Torrey, J. R. 2018. Pepper mild mottle virus as a water quality indicator. npj Clean Water 1:19.

Kwon, D. Y., Chung, K. R., Yang, H.-J. and Jang, D.-J. 2015. Gochujang (Korean red pepper paste): a Korean ethnic sauce, its role and history. J. Ethn. Foods 2:29-35.

Nakamura, S., Yang, C.-S., Sakon, N., Ueda, M., Tougan, T., Yamashita, A., Goto N., Takahashi, K., Yasunaga, T., Ikuta, K., Mizutani, T., Okamoto, Y., Tagami, M., Morita, R., Maeda, N., Kawai, J., Hayashizaki, Y., Nagai, Y., Horii, T., Iida, T. and Nakaya, T. 2009. Direct metagenomic detection of viral pathogens in nasal and fecal specimens using an unbiased high-throughtput sequencing approach. PLoS ONE 4:e4219.

Park, K.-Y., Kong, K.-R., Jung, K.-O. and Rhee, S.-H. 2001. Inhibitory effects of Kochujang extracts on the tumor formation and lung metastasis in mice. J. Food Sci. Nutr. 6:187-191.

Peng, J., Shi, B., Zheng, H., Lu, Y., Lin, L., Jiang, T., Chen, J. and Yan, F. 2015. Detection of pepper mild mottle virus in pepper sauce in China. Arch. Virol. 160:2079-2082.

Symonds, E. M., Rosario, K. and Breitbart, M. 2019. Pepper mild mottle virus: agricultural menace turned effective tool for microbial water quality monitoring and assessing (waste)water treatment technologies. PLoS Pathog. 15:e1007639.

Tamura, K., Peterson, D., Peterson, N., Stcher, G., Nei, M. and Kumar, S. 2011. MEGA5: molecular evolutionary genetics analysis using maximum likelihood, evolutionary distance, and maximum parsimony methods. Mol. Biol. Evol. 28:27312739.

Zhang, T., Breitbart, M., Lee, W. H., Run, J.-Q., Wei, C. L., Soh, S. W. L., Hibberd, M. L., Liu, E. T., Rohwer, F. and Ruan, Y. 2006. RNA viral community in human feces: prevalence of plant pathogenic viruses. PLoS Biol. 4:e3. 